\title{
Postpartum Weight Retention: A Retrospective Data Analysis Measuring Weight Loss and Program Engagement with a Mobile Health Program
}

\author{
Tatiana Toro-Ramos, $\mathrm{PhD},{ }^{1,2}$ Martica Heaner, $\mathrm{PhD},{ }^{3}$ Qiuchen Yang, MS, ${ }^{4}$ Laura DeLuca, MA, ${ }^{4,5}$ \\ Heather Behr, PhD, ${ }^{4}$ Kayla Reynolds, MS, ${ }^{6}$ Youngin Kim, MD, ${ }^{4,7}$ and Andreas Michaelides, $\mathrm{PhD}^{4}$
}

\begin{abstract}
Background: Mobile health (mHealth) technology can circumvent barriers to participation in weight loss programs faced by new mothers. The objective of this study was to assess weight change and program engagement in postpartum women $(n=130)$ participating in a 24 -week behavior change mHealth weight-loss intervention.

Materials and Methods: Participants were recruited through a program offered on a commercial mHealth application that provided evidence-based lifestyle interventions. To meet inclusion criteria, women had to be 18-45 years of age, and given birth within 2 years before the start of the study. Participants signed up for the Noom Healthy Weight program between January and March of 2019 and were offered the program free of charge. Linear mixed models were conducted; the primary outcome was weight change from baseline at 16 and 24 weeks. Secondary outcomes were program engagement and their relationship with completion status.

Results: Results showed that time was a significant predictor of weight at week $16[t(-3.94)=-9.40 ; p<0.001]$ and week $24[t(-4.08)=-9.74 ; p<0.001]$; users lost $3.94 \mathrm{kgs}$ at week 16 and $4.08 \mathrm{kgs}$ at week 24 , compared with baseline. In addition, body mass index significantly decreased at week $24[t(112)=7.33, p<0.0001]$ with the majority of participants $(80 \%)$ experiencing reductions by more than 2 units. On average, subjects who completed the program (completers) lost more weight compared with those who did not complete the program $[t(-5.09)=-2.94 ; p=0.004]$, losing $5.09 \mathrm{kgs}(95 \% \mathrm{CI}-8.48$ to -1.69$)$ throughout the 24 weeks.

Conclusion: This cohort study shows that a uniquely mobile, behavior change intervention for weight management is effective at producing significant weight loss with potential to address postpartum weight retention.
\end{abstract}

Keywords: postpartum, weight loss, mHealth, behavioral interventions, obesity

\section{Introduction}

A $S$ THE PREVALENCE of overweight and obesity has increased over the last four decades, not only has there been an increase in obesity-related comorbidities for individuals in the population, ${ }^{1,2}$ there have also been transgenerational effects, ${ }^{3}$ with the amount of weight gained during pregnancy influencing the future health of both the woman and her infant. ${ }^{4,5}$ The population demographics of women who become pregnant have changed dramatically over the past decade, and more women are overweight or obese at conception. In 2009, the Institute of Medicine (IOM) revised gestational weight gain $(\mathrm{GWG})$ guidelines that established more restrictive weight gain limitations based on prepregnancy body mass index (BMI). Women who fall within the overweight or obese BMI range begin pregnancies with an increased risk of excessive GWG, which can lead to complications during pregnancy and birth, ${ }^{6}$ increasing future health risks for both mother and child. ${ }^{7,8}$ Excessive weight gain during pregnancy has also been associated with

\footnotetext{
${ }^{1}$ Noom, Inc., New York, New York, USA.

${ }^{2}$ Project Management, Seed Health, Venice, California, USA.

${ }^{3}$ Nutrition Department, School of Urban Public Health, Hunter College, New York, New York USA.

${ }_{5}^{4}$ Clinical Research, Noom, Inc., New York, New York, USA.

${ }^{5}$ Ferkauf Graduate School of Psychology, Yeshiva University, Bronx, New York, USA.

${ }^{6}$ Curriculums, Noom, Inc., New York, New York, USA.

${ }^{7}$ Department of Biomedical Systems Informatics, Yonsei University College of Medicine Seoul, Seoul, South Korea.
}

(C) Tatiana Toro-Ramos et al. 2021; Published by Mary Ann Liebert, Inc. This Open Access article is distributed under the terms of the Creative Commons License (http://creativecommons.org/licenses/by/4.0), which permits unrestricted use, distribution, and reproduction in any medium, provided the original work is properly cited. 
postpartum weight retention (PPWR). ${ }^{9-11}$ This weight gain has shown to cause a cyclical effect where a woman enters her next pregnancy heavier compared with the previous, further increasing the risk of excessive GWG, greater subsequent PPWR, and poor health outcomes. ${ }^{10,12-14}$ Children born to heavier mothers have a higher risk of childhood and adult obesity and the related adverse health outcomes. ${ }^{7,15}$ Reducing excessive GWG and PPWR can help minimize this potential long-term weight trajectory, improving short- and long-term health risks for mother and child.

Within the literature, it has been suggested that, on average, women retain at least $4.5 \mathrm{~kg}$ at 1 year postpartum. ${ }^{12,13,16,17} \mathrm{~A}$ return to a healthy prepregnancy weight is considered optimal to reduce the risk of future obesity and adverse outcomes in subsequent pregnancies. Studies have shown that a combination of diet and exercise interventions postpartum can effectively reduce PPWR, ${ }^{8,11,18,20-22}$ although such interventions may not always be practical. Previous studies have highlighted the difficulty of engaging and maintaining contact with women in the postpartum period. ${ }^{18-20}$ Despite a desire to lose weight, engagement among this population is a challenge due to increased burdens such as family and work obligations and the pressures of raising a newborn child. ${ }^{18}$ Quantitative and qualitative studies have identified factors that make PPWR likely: Interventions may be costly and require (or be perceived to require) intensive participation that is not possible due to a lack of childcare, transportation limitations, and inflexible work schedules. ${ }^{23,24}$ Additionally, women have relayed that focusing on their own needs is not a priority and report excessive eating to relieve stress. ${ }^{25-27}$

The Academy of Nutrition and Dietetics (AND) recommends that new mothers have access to nutrition education and behavioral counseling to help reduce PPWR, especially during the first 12 to 18 months postpartum and during interconception. They acknowledge that attendance at external lifestyle intervention programs may be difficult, and technology tools, like text messages and online programs, may help adherence. ${ }^{10}$ Therefore, an optimal approach to address PPWR would be to overcome barriers of participation and adherence in this population. It has been noted that home-based interventions coupled with support from health professionals and modern technological tools (i.e., internet, text communications, and mobile apps) may be more effective than traditional structured community programs, which require traveling to in-person educational, coaching, or exercise sessions. ${ }^{13,20}$

An estimated 2.5 billion people own smartphones globally, according to the Pew Research Center, ${ }^{28}$ and behavioral health programs are taking advantage of this technology to deliver tailored lifestyle interventions. ${ }^{5,29,30}$ Mobile health (mHealth) may be especially helpful to the postpartum population as features associated with this technology offer a potential solution to the many barriers they encounter. Digital communications allow for the dissemination of education; informational and motivational content can be sent while participant engagement can be prompted through text messages, push notifications, email, or social media posts. In addition, mHealth enables easy selfmonitoring of food intake and exercise, alongside other program variables. A systematic review of previous postpartum mHealth interventions concluded that woman lost an average of $-2.55 \mathrm{~kg}$ (95\% CI -3.81 to -1.28 ) with interventions lasting from 23 to 52 weeks. ${ }^{5}$ However, more is needed to understand the overall efficacy of mHealth interventions in this population.
The objective of this cohort study was to assess short-term weight loss among postpartum women and their level of engagement with a variety of features in a mHealth behavior change program. We hypothesized time would be predictive of weight at 16 and 24 weeks, such that women in the mHealth intervention would lose weight over time. We also predicted that completion status of the program would predict weight; completers would lose more weight compared with starters and nonstarters.

\section{Materials and Methods}

\section{Participants}

Participants were recruited through a program offered on a commercial mHealth application that provided evidencebased lifestyle interventions. To meet inclusion criteria, women had to be 18-45 years of age, who had given birth within 2 years before the start of the study. These participants signed up for the Noom Healthy Weight program between January and March of 2019 and were offered the program free of charge. Women currently pregnant or who had given birth more than 2 years before study entry were excluded. This study, including screening questions and informed consent, were approved by an external IRB. All participants in the study reviewed and signed the approved informed consent before participation.

\section{Design}

This was a retrospective data analysis examining weight change and behavioral outcomes of postpartum women who enrolled in the interventional program.

\section{Weight loss intervention}

Upon sign up, participants were prompted to enter their height, weight, and weight loss goal. Throughout the first week, participants received digital instructions on how to follow the Healthy Weight program through the Noom platform on their mobile devices (Fig. 1). The Noom program features an interactive interface providing virtually delivered Cognitive Behavior Therapy techniques, including coach/participant messaging, group messaging, daily challenges to improve habits, educational articles, and meal tracking. Participants were instructed to read articles, log food and exercise, and to interact with a personal coach, as well as other participants in a group format.

The structure of the intervention was kept purposefully flexible as the emphasis was aimed at establishing a system of healthy living that could be adopted long term for lasting lifestyle change. Therefore, the objective was for each participant to interact with the program, including the coach and the group, in a way that was smoothly integrated into their normal lifestyle patterns. Participants were not required to $\log$ a designated amount of meals or to perform a specific amount of activity. Instead, participants were provided with one to three daily educational articles, which included nutritional and motivational information to guide their individual choices of their meals and exercise. Participants were instructed to communicate through the mobile program for support and $\log$ their food choices and exercise behaviors as often as possible. A pedometer embedded in the Noom mobile intervention tracked steps. Web-based coaches monitored participant progress and assisted in weekly goal setting through a secure dashboard. 

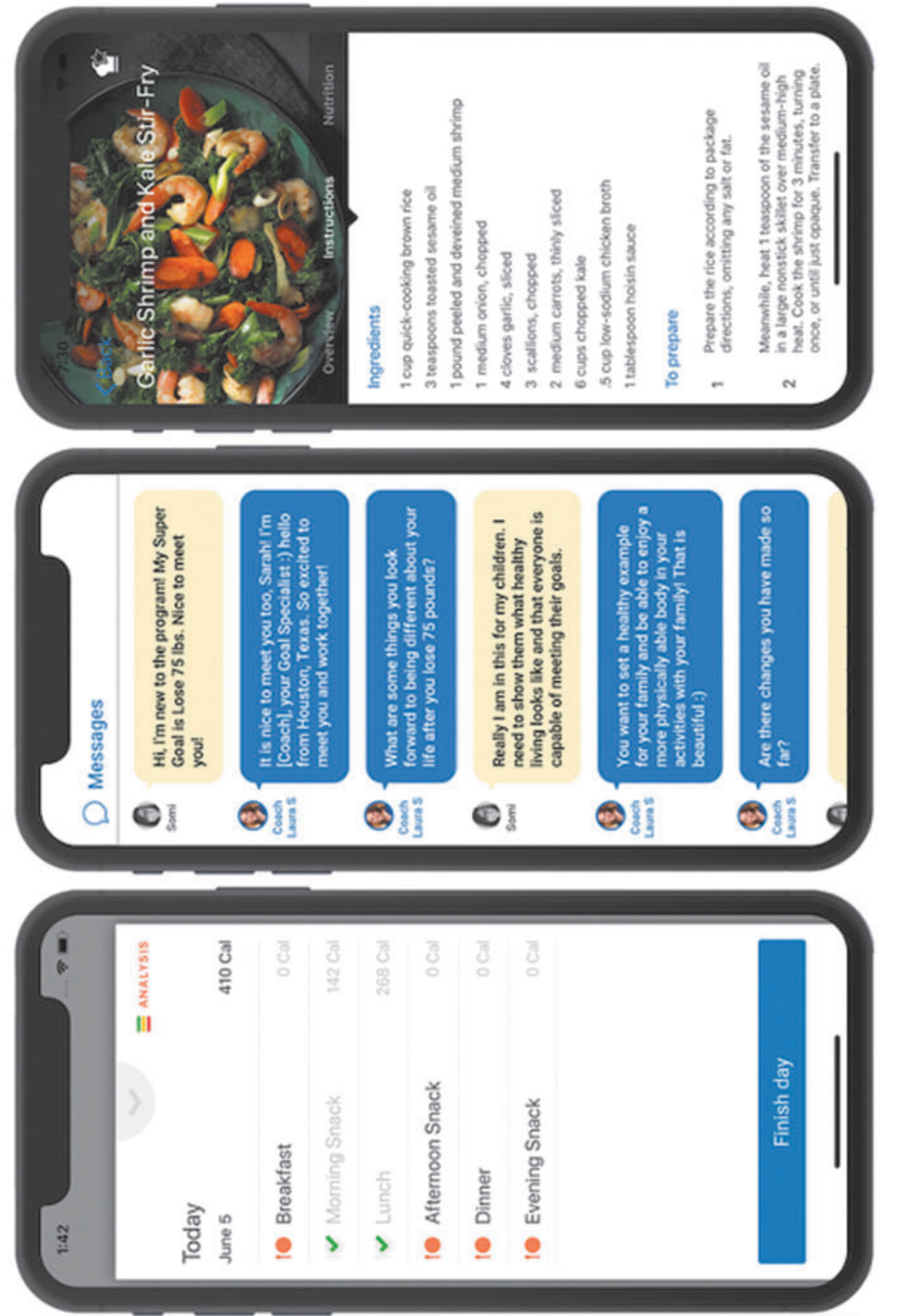

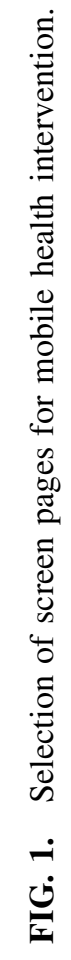

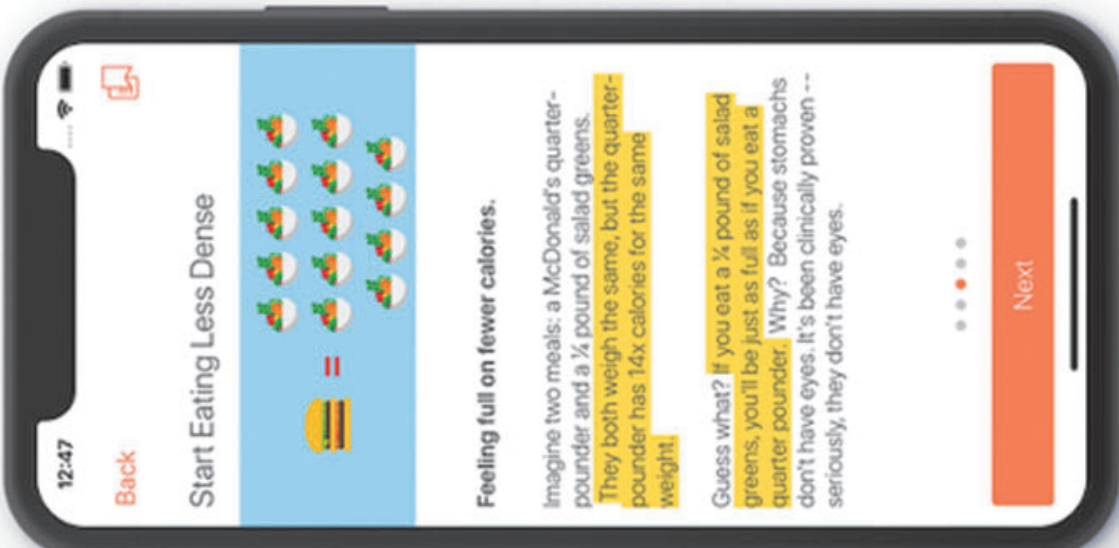




\section{Outcome measures}

The primary outcome under investigation was weight at 16 and 24 weeks. Self-reported height and weight were recorded within the program upon sign-up. Recommendations at the start of the program directed participants to log their weight at a set time each day, which appeared in a list of daily tasks. The secondary outcome explored was engagement with inprogram features and the association with completion status. Article engagement, that is, the rate of article reading, has been shown to be predictive of overall participation among previous users of this program. ${ }^{31,32}$ Additional forms of engagement were observed and included: Self-reported measures of the number of meals logged per week, exercise sessions logged, weigh-ins, and total minutes exercising, and behavioral-based measures extracted from the program: group posts, group comments, messages to the coach, group likes, and steps recorded and time spent exercising.

Based on the CDC's definition for "attendance" in their Diabetes Prevention Program, ${ }^{33}$ participants were considered program "starters" if they read at least one article per week for at least 4 weeks. Participants were "completers" if they read at least one article per week over 9 weeks out of 16 core program weeks and communicated with the coach at least once per week over 3 weeks. "Nonstarters", were participants who read less than one article per week over 3 weeks and did not engage in any additional part of the program.

\section{Statistical analyses}

Descriptive statistics, expressed as means and standard deviations (SDs) or frequencies and percentages for continuous and categorical variables, were calculated for subject baseline characteristics. Linear mixed-effects models evaluated changes in our primary outcome of weight. The benefit of using linear mixed-effects models is that it accounts for missing data both at random and not at random. ${ }^{34}$ All users in the program were analyzed despite any missingness related to completion status; weight outcomes were predicted from the linear mixed models conducted. Time (Baseline, week 16, week 24) and completion status (nonstarters, starters, completers) were both conceptualized as a three-level categorical variable. An interaction between time and engagement was not included in the model, given the issue of multicollinearity. Fixed effects included time, completion status, age, and baseline BMI. The intercept for each participant was included as random effects to account for the within-subject correlation. When age was added into the model, we found that the model was not significantly improved based on the chi-square goodness-of-fit test $\left(\chi^{2}=0.06, p=0.80\right)$, therefore age was not significantly predicting the weight. However, age was found as a significant predictor in our previous study, therefore, we decided to keep age in our final model. An exchangeable covariance matrix yielded the best fit model for the repeated effect of time, using visual inspection and Akaike's information Criteria. Paired $t$-tests were used to compare the differences in participants' BMI between preintervention and postintervention. Mean weekly engagement variables were calculated as means and SDs from baseline to 24 weeks. Last observation carried forward was utilized for endpoints. Significance tests were two tailed with a significance of $p<0.05$. Statistical analyses were performed using $\mathrm{R}$ version 3.6.0 software.

\section{Results}

Demographic characteristics of participants stratified by their completion status are shown in Table 1. Of the 130 participants, 90 (69\%) of them actively participated: 33 (25\%) met "starters" criteria and 57 (44\%) met "completers" criteria. Forty (31\%) were considered "nonstarters" since they exhibited a minimal level of participation $(<1$ article read per week in the first 3 weeks and no other engagement). Women were, on average, $32.1(\mathrm{SD}=4.77)$ years old, 40.9 ( $\mathrm{SD}=27.54)$ weeks postpartum and classified as having obesity with a mean BMI of 33.2 ( $\mathrm{SD}=6.98)$. At baseline, completers were significantly older than nonstarters by 2.4 years $[33.4(\mathrm{SD}=4.72)$ vs. $31.0(\mathrm{SD}=4.99), t(112)=$ 7.3, $p=0.033]$.

Our findings indicated that mean weight loss declined steadily through week 16 then exhibited a slowdown up to week 24 (Fig. 2). Results for the paired $t$-tests showed that on average, BMI decreased at week $24 \quad[t(112)=7.33$, $p<0.0001]$ with a majority of participants (80\%) experiencing significant reductions: $31 \%$ decreased BMI by more than 2 units, and $9 \%$ by more than 5 units. Approximately $19 \%$ of women who were classified by BMI as overweight at baseline shifted to normal range by week 24 (Table 2). Thirty-nine percent of women classified as having obesity at baseline dropped to the overweight classification; $8 \%$ of those with Class 2 obesity (35.0-39.9 BMI) reached overweight status by week 24 . When evaluating weight over time, adjusting for baseline BMI, age, and completion status, time at 16 weeks $[t(-3.94)=-9.40 ; p<0.001$; Table 3$]$ and time at 24 weeks $[t(-4.08)=-9.74 ; p<0.001$; Table 3] were both significant predictors of weight. Compared with baseline, participants on average, regardless of completion status, lost $-3.94 \mathrm{~kg}$ at week 16 and $-4.08 \mathrm{~kg}$ at week 24 . Completion status was found to be predictive of weight, where completers

Table 1. Baseline Characteristics of Study Nonstarters, Starters, and Completers

\begin{tabular}{lcccc}
\hline & $\begin{array}{c}\text { All }(\mathrm{N}=130), \\
\text { mean } \pm S D\end{array}$ & $\begin{array}{c}\text { Nonstarters }(\mathrm{N}=40), \\
\text { mean } \pm S D\end{array}$ & $\begin{array}{c}\text { Starters }(\mathrm{N}=33), \\
\text { mean } \pm S D\end{array}$ & $\begin{array}{c}\text { Completers }(\mathrm{N}=57), \\
\text { mean } \pm S D\end{array}$ \\
\hline Mean age, years & $32.13 \pm 4.77$ & $31.00 \pm 4.99 *$ & $31.24 \pm 4.09$ & $33.44 \pm 4.72 *$ \\
Height, m & $1.66 \pm 0.07$ & $1.66 \pm 0.07$ & $1.67 \pm 0.08$ & $1.65 \pm 0.07$ \\
Weight, kg & $91.11 \pm 20.51$ & $94.45 \pm 25.24$ & $90.19 \pm 21.84$ & $89.31 \pm 15.53$ \\
BMI, kg/m & $33.18 \pm 6.98$ & $34.11 \pm 8.03$ & $32.54 \pm 7.75$ & $32.91 \pm 5.66$ \\
Time postpartum, weeks & $40.90 \pm 27.54$ & $39.18 \pm 25.48$ & $43.74 \pm 29.20$ & $40.38 \pm 28.22$ \\
\hline
\end{tabular}

${ }^{*} p=0.033$.

BMI, body mass index; SD, standard deviation. 
lost $5.09 \mathrm{~kg}$ more than nonstarters throughout the entire program $[t(-5.09)=-2.94 ; p=0.004$; Table 3]. Comparing starters to nonstarters, starters showed no difference in weight throughout the program $(0.29 \mathrm{~kg} ; 95 \% \mathrm{CI}-3.48$ to 4.07). On average, compared with starters, program completers read 3.6 more articles (15.01 read), weighed-in 3.5 more times (3.97 weigh ins), and logged 3.8 times more meals (12.06 meals logged) per week. Completers' exercise duration was 11.2 times longer (37.10 minutes) per week than starters; they had registered 2.33 times more steps per week (30,441 steps), made 4.8 more group posts per week $(0.32)$, and communicated with their coach 2.8 times as much per week (1.98 messages) compared with starters (Table 4).

\section{Discussion}

The current study explored weight loss and program engagement for postpartum women using a mHealth weight loss intervention. To our knowledge, this is the first study to observe this population in a fully mobile, behavior change lifestyle intervention. In support of our hypothesis, time (i.e., the weight loss trajectory) was a significant predictor of weight at weeks 16 and 24. Postpartum women in our cohort study, on average, lost 3.94 and $4.08 \mathrm{kgs}$ at weeks 16 and 24, respectively. While no formal guidelines have been set to define clinically meaningful postpartum weight loss, studies have shown that on average, women retain at least $4.5 \mathrm{~kg}$ at 1 year postpartum. ${ }^{13}$ The 2009 IOM report examined 1 cohort of 49,000 women and found that one-half had retained $>4.5 \mathrm{~kg}$ and one-quarter $>9 \mathrm{~kg}$ at 24 weeks postpartum. Another sample of 4,000 women found that one-quarter retained $>4.5 \mathrm{~kg}$ and $12 \%>9 \mathrm{~kg}$ at 1 year $(16,20)$. Another study looking at 774 women found they retained an average of $5 \mathrm{~kg}$ 1 year postpartum and $75 \%$ weighed more than they did

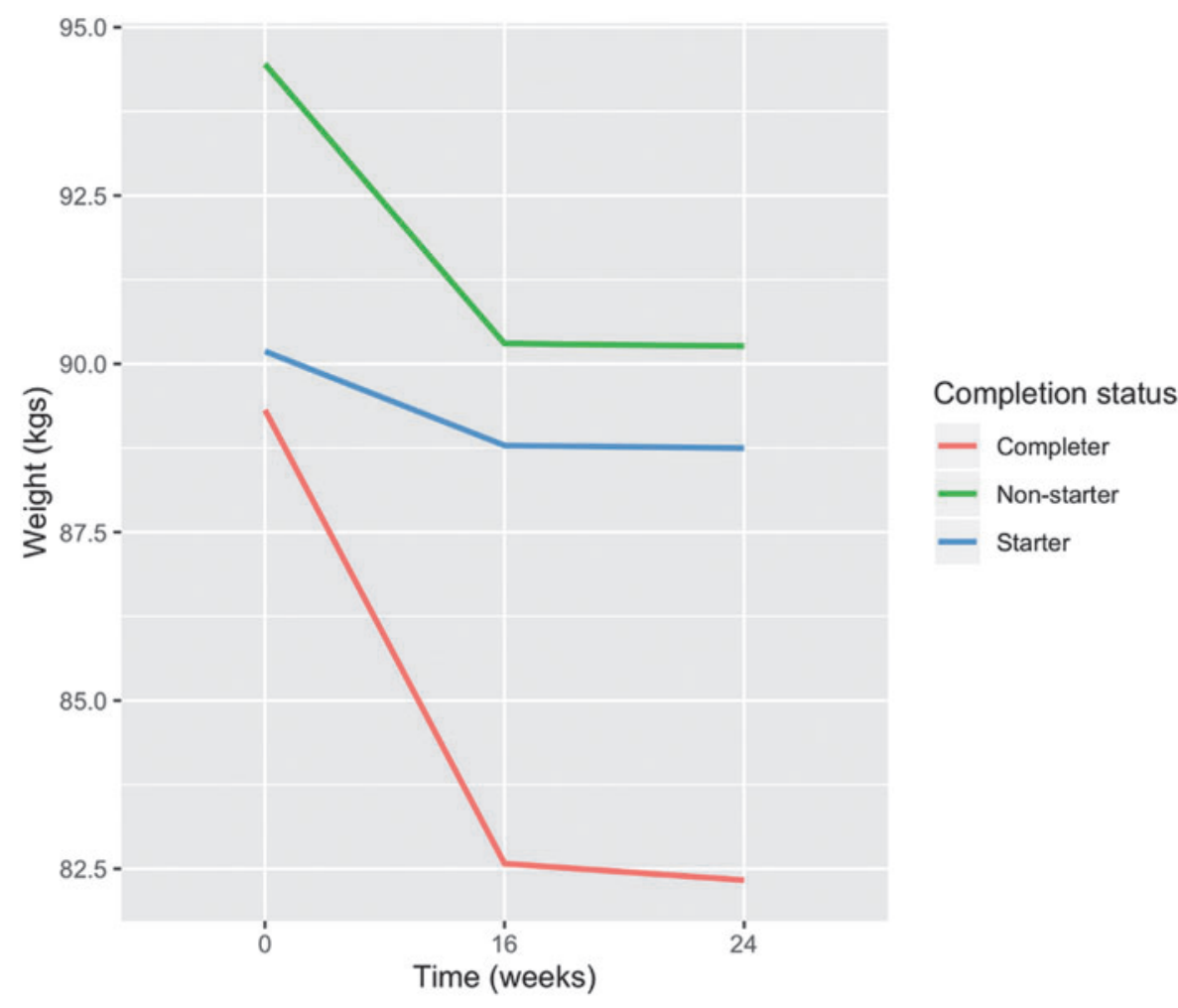

prepregnancy. ${ }^{12}$ Thus our findings, compared with CDC guidelines, ${ }^{36}$ suggest our intervention can serve as an appropriate and successful weight loss method for postpartum women. In addition, completion status was found to be predictive of weight, where participants who completed the program lost $5.09 \mathrm{kgs}$ more than nonstarters, which is in support of our secondary hypothesis. These results suggest that, if maintained, participants who actively engage in a similar mHealth postpartum intervention can successfully reduce weight retention and improve BMI status.

A strength of this study was that the platform used was entirely mobile. It has been reported that minority and lowincome households are the most frequent users of mobile phones for Internet access. ${ }^{37}$ Hence, this type of intervention may be ideal for overcoming barriers of participation and adherence in this population. Moreover, this study included a unique protocol: Women were not assigned a specific diet, exercise plan, or set of rules to follow. They were provided with educational information to decide which healthy behaviors they would implement, along with motivational support and goal setting from their online coach. Future research is needed to understand the effectiveness of programs that provide a structured (prescriptive) program compared with an unstructured cognitive behavior-based program offering participant support to determine if one provides more benefit over the other.

However, caution must be made in extrapolating participants' behaviors into a specific lifestyle intervention prescription. Level of engagement may be more predictive of weight loss than which diet or exercise protocol was followed. For example, the program pedometer registered only 30,441 steps per week, or $\sim 4,349$ steps per day, the equivalent to what a sedentary office worker might obtain and is considered a suboptimal amount for weight management.
FIG. 2. Mean weight loss across time. 
Table 2. Body Mass Index Categories at Baseline and at 24 Weeks

\begin{tabular}{|c|c|c|c|c|c|}
\hline & \multicolumn{5}{|c|}{ BMI categories at 24 weeks ${ }^{\mathrm{a}}$} \\
\hline & $18.5-24.9$ & $25.0-29.9$ & $30.0-34.9$ & $35.0-39.9$ & $>40$ \\
\hline & $\begin{array}{l}\text { Normal, } \\
\mathrm{n}(\%)\end{array}$ & $\begin{array}{l}\text { Overweight, } \\
\mathrm{n}(\%)\end{array}$ & $\begin{array}{c}\text { Obesity (Class I), } \\
\text { n }(\%)\end{array}$ & $\begin{array}{c}\text { Obesity (Class II), } \\
\mathrm{n}(\%)\end{array}$ & $\begin{array}{l}\text { Extreme obesity } \\
\text { (Class III), n (\%) }\end{array}$ \\
\hline \multicolumn{6}{|l|}{ BMI categories at baseline } \\
\hline $18.5-24.9$ & $10(91)$ & $1(9)$ & $0(0)$ & $0(0)$ & $0(0)$ \\
\hline \multicolumn{6}{|l|}{ Normal } \\
\hline $25.0-29.9$ & $6(19)$ & $23(74)$ & $2(7)$ & $0(0)$ & $0(0)$ \\
\hline \multicolumn{6}{|l|}{ Overweight } \\
\hline $30.0-34.9$ & $0(0)$ & $13(39)$ & $20(61)$ & $0(0)$ & $0(0)$ \\
\hline \multicolumn{6}{|l|}{ Obesity (Class I) } \\
\hline $35.0-39.9$ & $0(0)$ & $2(8)$ & $8(32)$ & $14(56)$ & $1(4)$ \\
\hline \multicolumn{6}{|l|}{ Obesity (Class II) } \\
\hline$>40$ & $0(0)$ & $0(0)$ & $0(0)$ & $3(23)$ & $10(77)$ \\
\hline Extreme obesity (Class III) & & & & & \\
\hline
\end{tabular}

${ }^{\mathrm{a}} p<0.001$.

Current guidelines recommend that women postpartum perform at least 150 minutes of moderate-intensity activity per week and for weight management (in all adults), up to 300 minutes or more per week, with daily exercise considered optimal. ${ }^{38,39}$ Completers only reported an average of 2.25 exercise sessions for only 37 minutes per week (Table 4). These metrics appear to show an inadequate level of commitment as it is well established that more exercise is required for measurable weight loss. Therefore, it is likely that participants did more physical activity than reported. Thus, the logging shown in the program reflects the action of logging, but not the total amount of activity performed. In an in-house survey of Noom lifestyle coaches (not shown), 94\% (100/ 106) confirmed that many participants relay through text communications that they have performed more exercise than they are logging into the program. Additionally, the pedometer will only track movement while the phone is being held and external physical activity monitoring devices may not have been synced to the program. Similarly, meals logged

Table 3. Summary of Linear Mixed Effects MODEL RESUlts

\begin{tabular}{|c|c|c|c|c|}
\hline Random effects & Variance & $\begin{array}{l}\text { Standard } \\
\text { deviation }\end{array}$ & & \\
\hline $\begin{array}{l}\text { Participant ID } \\
\text { (intercept) }\end{array}$ & 61.95 & 7.87 & & \\
\hline Fixed effects & Estimate & Standard Error & $\mathrm{t}$ & $\mathrm{p}$ \\
\hline BMI & 2.60 & 0.10 & 24.94 & 0.001 \\
\hline Age & -0.04 & 0.16 & -0.25 & 0.80 \\
\hline \multicolumn{5}{|l|}{ Time } \\
\hline Baseline & - & - & - & - \\
\hline 16 weeks & -3.94 & 0.42 & -9.40 & $<0.001$ \\
\hline 24 weeks & -4.08 & 0.42 & -9.74 & $<0.001$ \\
\hline \multicolumn{5}{|c|}{ Completion status } \\
\hline Nonstarters & - & - & - & - \\
\hline Starters & 0.29 & 1.92 & 0.15 & 0.88 \\
\hline Completers & -5.09 & 1.73 & -2.90 & $<0.01$ \\
\hline
\end{tabular}

Baseline and nonstarters are used as the reference group. $p<0.05$. Estimate represents predicted value of weight. were fewer than two per day (12.06/week). Without complete energy intake information, it is not possible to establish clear recommendations about what aspects of diet contributed to successful weight loss.

While it would be useful to obtain exact amounts and frequencies of exercise and amounts and types of food consumed to determine the optimal food and fitness prescriptions likely to produce favorable weight loss results, which was not the aim. The goal of the study was to motivate participants by using a variety of tools to keep them proactive in making healthier food choices and becoming physically active enough to reduce PPWR. Future research is needed to determine ways to account for unregistered information both when designing interventions and in future program development. This study indicates that weight loss can be achieved when following a self-determined plan and that participant completion status is predictive of weight loss. Additionally, this study demonstrated that a mHealth tool for behavior change paired with a virtual coach can keep participants focused and motivated to obtain weight loss results.

\section{Limitations}

This study is not without limitations. Given the nature of our observational study design, the effect of the intervention against a control group is unknown. Therefore caution is warranted when interpreting the results. Second, our outcome measure is based on self-reported data and may not represent true measures of activity or food behaviors. Last, baseline characteristics do not include additional demographic and other personal information, including ethnicity, income levels, breastfeeding, BMI status before pregnancy, and amount of GWG that may have been associated with PPWR. 11,37,40,41 For privacy reasons, much of this information is not collected for the average Noom program user. The determinants and barriers that affect postpartum weight loss interventions, as well as the specific prescriptions that create weight loss in this population are encouraged in further studies.

Based on our findings, weight loss declined steadily through week 16 then exhibited a slowdown up to week 24 (Fig. 2). It is not uncommon to see a plateauing effect, although typically it is reported at around 6 months. ${ }^{38}$ This 
Table 4. Mean Weekly Program Engagement Among Nonstarters, Starters, and Completers Over Week 24

\begin{tabular}{lcccc}
\hline & All $(\mathrm{n}=130)$ & $\begin{array}{c}\text { Nonstarters } \\
(\mathrm{n}=40)\end{array}$ & $\begin{array}{c}\text { Starters } \\
(\mathrm{n}=33)\end{array}$ & $\begin{array}{c}\text { Completers } \\
(\mathrm{n}=57)\end{array}$ \\
\hline Meals logged (meals per week) & $7.21 \pm 6.41$ & $1.07 \pm 0.92$ & $3.16 \pm 1.88$ & $12.06 \pm 5.53$ \\
Exercise (times/week) & $1.43 \pm 2.25$ & $0.14 \pm 0.33$ & $0.94 \pm 1.60$ & $2.25 \pm 2.66$ \\
Time exercised (minutes/week) & $20.13 \pm 45.05$ & $2.22 \pm 4.02$ & $3.30 \pm 8.57$ & $37.10 \pm 59.47$ \\
Steps recorded (steps/week) & $20,493.70 \pm 18,662.21$ & $6,698.78 \pm 7,386$ & $13,080 \pm 9,850.57$ & $30,441.06 \pm 20,175.58$ \\
Number of weigh-ins (times/week) & $2.43 \pm 2.08$ & $0.46 \pm 0.38$ & $1.14 \pm 0.67$ & $3.97 \pm 1.83$ \\
Articles read (articles/week) & $7.86 \pm 7.79$ & $1.50 \pm 0.90$ & $4.22 \pm 2.58$ & $15.01 \pm 6.72$ \\
Group posts (posts/week) & $0.17 \pm 0.48$ & $0.00 \pm 0.02$ & $0.07 \pm 0.15$ & $0.32 \pm 0.64$ \\
Group comments (comments/week) & $0.51 \pm 0.97$ & $0.01 \pm 0.32$ & $0.17 \pm 0.22$ & $0.91 \pm 1.23$ \\
Messages to coach (messages/week) & $1.26 \pm 1.19$ & $0.30 \pm 0.24$ & $0.72 \pm 0.44$ & $1.98 \pm 1.28$ \\
Group likes (likes/week) & $0.40 \pm 0.82$ & $0.01 \pm 0.17$ & $0.12 \pm 0.23$ & $0.73 \pm 1.05$ \\
\hline
\end{tabular}

could be attributed to physiological adaptations to weight $\operatorname{loss}^{39}$ and/or a reduction in motivation and adherence to an intervention. In this case, it likely reflected a reduction in treatment intensity as the core program in the Healthy Weight intervention is 16 weeks long. While the reduction phase can be extended, most participants stopped or reduced engagement before continuing in the maintenance phase from weeks 16 to 24 . Because a majority of participants still qualified as completers, their week 16 observations were estimated, therefore not exhibiting their true weight loss.

\section{Conclusion}

Postpartum women who completed a unique mHealth program, based on cognitive behavioral therapy, lost significant weight at week 24 . This study demonstrated that time and completion of the program, which included engagement with the online coach, was predictive of weight loss. In conclusion, this study demonstrates the effectiveness of an intervention targeting PPWR that is delivered entirely through a smartphone platform with an unstructured approach which allowed participants to self-select the diet and exercise protocol. Future digital intervention studies should follow postpartum women over longer periods, and account for breastfeeding and sleep quantity, and include robust body composition measures to help inform the development of more tailored interventions for such an important lifecycle window.

\section{Acknowledgments}

The authors would like to thank all study participants for their important contribution to this study. For statistics guidance and expertise, they are grateful to Dr. David González Barreto, PhD at the University of Puerto Rico, Mayagüez. They would also like to acknowledge Alissa Carey, MA, who participated in writing, proofreading, and technical preparation of the article.

Deidentified participant data collected for the study will be made available to bona fide researchers, subject to a nondisclosure agreement on agreement by both parties after approval of a methodologically sound proposal. Upon approval, we will provide a minimal dataset with deidentified participant data and data dictionary up to 1 year after publication to achieve the aims in the proposal. The study's protocol will also be made available upon request as part of the proposal. Proposals should be directed to the corresponding author at research@noom.com

\section{Author Disclosure Statement}

L.D., Q.Y., H.B., K.R., Y.K., and A.M. are employed by Noom, Inc., and receive a salary and stock options. A.M. holds a patent pending (3492.004US1) with Noom, Inc., Noom Coach is a mobile application owned by Noom, Inc., The authors have no additional conflicts of interest, and no competing financial interests exist for the other authors.

\section{Funding Information}

No funding was received for this article.

\section{References}

1. Andolfi C, Fisichella PM. Epidemiology of obesity and associated comorbidities. J Laparoendosc Adv Surg Tech A 2018;28:919-924.

2. Rowley WR, Bezold C, Arikan Y, Byrne E, Krohe S. Diabetes 2030: Insights from yesterday, today, and future trends. Popul Health Manag 2017;20:6-12.

3. Haire-Joshu D, Tabak R. Preventing obesity across generations: Evidence for early life intervention. Annu Rev Public Health 2016;37:253-271.

4. Cnattingius S, Villamor E, Lagerros YT, Wikstrom AK, Granath F. High birth weight and obesity-A vicious circle across generations. Int J Obes (Lond) 2012;36:1320-1324.

5. Sherifali D, Nerenberg KA, Wilson S, Semeniuk K, et al. The effectiveness of eHealth technologies on weight management in pregnant and postpartum women: Systematic review and meta-analysis. J Med Internet Res 2017;19:e337.

6. Gould Rothberg BE, Magriples U, Kershaw TS, Rising SS, Ickovics JR. Gestational weight gain and subsequent postpartum weight loss among young, low-income, ethnic minority women. Am J Obstet Gynecol 2011;204:52-e1.

7. ACOG. Weight gain during pregnancy. Committee Opinion No. 548. American College of Obstetricians and Gynecologists. Obstet Gynecol 2013;121:210-213.

8. Farpour-Lambert NJ, Ells LJ, Martinez de Tejada B, Scott C. Obesity and weight gain in pregnancy and postpartum: An evidence review of lifestyle interventions to inform maternal and child health policies. Front Endocrinol (Lausanne) 2018;9:546.

9. Rong K, Yu K, Han X, Szeto IM, et al. Pre-pregnancy BMI, gestational weight gain and postpartum weight retention: A meta-analysis of observational studies. Public Health Nutr 2015;18:2172-2182.

10. Stang J, Huffman LG. Position of the Academy of Nutrition and Dietetics: Obesity, reproduction, and pregnancy outcomes. J Acad Nutr Diet 2016;116:677-691. 
11. Choi J, Fukuoka Y, Lee JH. The effects of physical activity and physical activity plus diet interventions on body weight in overweight or obese women who are pregnant or in postpartum: A systematic review and meta-analysis of randomized controlled trials. Prev Med 2013;56:351-364.

12. Endres LK, Straub H, McKinney C, et al. Postpartum weight retention risk factors and relationship to obesity at 1 year. Obstet Gynecol 2015;125:144.

13. McKinley MC, Allen-Walker V, McGirr C, Rooney C, Woodside JV. Weight loss after pregnancy: Challenges and opportunities. Nutr Res Rev 2018;31:225-238.

14. Lipsky LM, Strawderman MS, Olson CM. Maternal weight change between 1 and 2 years postpartum: The importance of 1 year weight retention. Obesity (Silver Spring) 2012;20: 1496-1502.

15. O'Reilly JR, Reynolds RM. The risk of maternal obesity to the long-term health of the offspring. Clin Endocrinol (Oxf) 2013;78:9-16.

16. Rasmussen KM, Yaktine AL. Institute of Medicine and National Research Council Committee to Reexamine IOM Pregnancy Weight Guidelines (2009) Weight Gain During Pregnancy: Reexamining the Guidelines. Washington, DC: National Academies Press, 2009.

17. Headen IE, Davis EM, Mujahid MS, Abrams B. Racialethnic differences in pregnancy-related weight. Adv Nutr 2012;3:83-94.

18. Dodd JM, Deussen AR, O'Brien CM, et al. Targeting the postpartum period to promote weight loss: A systematic review and meta-analysis. Nutr Rev 2018;76:639-654.

19. Linné Y, Dye L, Barkeling B, Rössner S. Long-term weight development in women: A 15-year follow-up of the effects of pregnancy. Obes Res 2004;12:1166-1178.

20. van der Pligt $\mathrm{P}$, Willcox J, Hesketh KD, Ball K, Wilkinson $\mathrm{S}$, et al. Systematic review of lifestyle interventions to limit postpartum weight retention: Implications for future opportunities to prevent maternal overweight and obesity following childbirth. Obes Rev 2013;14:792-805.

21. Dalrymple KV, Flynn AC, Relph SA, O'Keeffe M, Poston L. Lifestyle interventions in overweight and obese pregnant or postpartum women for postpartum weight management: A systematic review of the literature. Nutrients 2018;10:1704.

22. Nascimento SL, Pudwell J, Surita FG, Adamo KB, Smith GN. The effect of physical exercise strategies on weight loss in postpartum women: A systematic review and metaanalysis. Int J Obes (Lond) 2014;38:626-635.

23. Silfee VJ, Lopez-Cepero A, Lemon SC, Estabrook B, Nguyen O, Rosal MC. Recruiting low-income postpartum women into two weight loss interventions: In-person versus Facebook delivery. Transl Behav Med 2019;9:129-134.

24. Santos KD, Patricio PT, Lima TSV, Barros DC, Saunders C. A pilot intervention to reduce postpartum weight retention at primary health care in Brazil. Nutr Hosp 2019;36: 854-861.

25. Christenson A, Johansson E, Reynisdottir S, Torgerson J, Hemmingsson E. Women's perceived reasons for their excessive postpartum weight retention: A qualitative interview study. PLoS One 2016;11:e0167731.

26. Faria-Schutzer DB, Surita FG, Rodrigues L, Turato ER. Eating behaviors in postpartum: A qualitative study of women with obesity. Nutrients 2018;10:885.

27. Murray-Davis B, Grenier L, Atkinson SA, et al. Experiences regarding nutrition and exercise among women during early postpartum: A qualitative grounded theory study. BMC Pregnancy Childbirth 2019;19:368.
28. Silver L. Smartphone ownership is growing rapidly around the world, but not always equally. Pew Research Center 2019 [updated February 5-November 15]. Available at: https://www.pewresearch.org/global/2019/02/05/smartphoneownership-is-growing-rapidly-around-the-world-but-notalways-equally Accessed May 10, 2019.

29. Lee JA, Choi M, Lee SA, Jiang N. Effective behavioral intervention strategies using mobile health applications for chronic disease management: A systematic review. BMC Med Inform Decis Mak 2018;18:12.

30. Harari GM, Lane ND, Wang R, Crosier BS, Campbell AT, Gosling SD. Using smartphones to collect behavioral data in psychological science: Opportunities, practical considerations, and challenges. Perspect Psychol Sci 2016;11:838-854.

31. Kim H, Ray CD, Veluscek AM. Complementary support from facilitators and peers for promoting mHealth engagement and weight loss. J Health Commun 2017;22:905-912.

32. Michaelides A, Major J, Pienkosz E, Jr., Wood M, Kim Y, Toro-Ramos T. Usefulness of a novel mobile diabetes prevention program delivery platform with human coaching: 65-week observational follow-up. JMIR Mhealth Uhealth 2018;6:e93.

33. Centers for Disease Control and Prevention. Centers for disease control and prevention diabetes prevention recognition program: Standards and operating procedures. Atlanta, GA: Centers for Disease Control and Prevention, 2015.

34. Mallinckrodt C, Clark WS, David S. Accounting for dropout bias using mixed-effects models. J Biopharm Stat 2001;11:9-21.

35. DeLuca L, Toro-Ramos T, Michaelides A, Seng E, Swencionis C. Does Age predict weight loss using Noom: A behavior change lifestyle intervention? JMIR Diabetes 2020;5:e18363.

36. CDC. CDC Diabetes Prevention Recognition Program: Standards and Operating Procedures. 2018. https://www .cdc.gov/diabetes/prevention/pdf/dprp-standards.pdf Accessed May 10, 2019.

37. Phelan S, Hagobian T, Brannen A, et al. Effect of an internet-based program on weight loss for low-income postpartum women: A randomized clinical trial. JAMA 2017;317:2381-2391.

38. Donnelly JE, Blair SN, Jakicic JM, Manore MM, Rankin JW, Smith BK. Appropriate physical activity intervention strategies for weight loss and prevention of weight regain for adults. Med Sci Sports Exerc 2009;41:459-471.

39. Magkos F, Fraterrigo G, Yoshino J, et al. Effects of moderate and subsequent progressive weight loss on metabolic function and adipose tissue biology in humans with obesity. Cell Metab 2016;23:591-601.

40. Gilmore LA, Klempel MC, Martin CK, et al. Personalized mobile health intervention for health and weight loss in postpartum women receiving women, infants, and children benefit: A randomized controlled pilot study. J Womens Health (Larchmt) 2017;26:719-727.

41. Jarlenski MP, Bennett WL, Bleich SN, Barry CL, Stuart EA. Effects of breastfeeding on postpartum weight loss among U.S. women. Prev Med 2014;69:146-150.

Address correspondence to: Andreas Michaelides, PhD Clinical Research Noom, Inc.

229 West 28th Street, Floor 9 New York, NY 10001

USA

E-mail: andreas@noom.com 